\title{
MEDICINA GENÓMICA EN LAS ESCUELAS DE MEDICINA DEL PERÚ
}

\author{
GENOMIC MEDICINE IN THE SCHOOLS OF MEDICINE OF PERU \\ César Ñique-Carbajal1,a, Josely Pérez-Loaiza'b, , Jeanette Mestanza-Quispe ${ }^{1, b}$
}

\begin{abstract}
Sr. Editor
La medicina genómica juega hoy en día un papel importante en la promoción de la salud de la poblaciones, esta se define como el uso de la información y tecnologías genómicas para determinar el riesgo, predisposición, diagnóstico y pronóstico de las enfermedades, con enfoque personalizado de las opciones terapéuticas. En definitiva es el uso de la información genotípica de un paciente en beneficio de su atención clínica ${ }^{(1,2)}$.

En el Perú, son pocas las instituciones que se encuentran adecuadamente equipadas e implementadas para desarrollar tanto actividades de genética como de genómica aplicada a la práctica médica, los esfuerzos que se vienen ejecutando son en tópicos puntuales y de una manera poco sostenible. Estos temas abarcan enfermedades neurológicas, cáncer hereditario, farmacogenética y algunos temas pediátricos. Asimismo, la investigación peruana en estas áreas emerge a paso lento, con instituciones representativas que se perfilan en dicho tema, entre las que podemos mencionar: Centro de Investigación de Genética y Biología Molecular de la Universidad San Martín de Porres, los Laboratorios de Investigación y Desarrollo de Ciencia y Tecnología de la Universidad Peruana Cayetano Heredia, el Instituto de Investigación en Ciencias Biomédicas de la Universidad Ricardo Palma, asimismo como parte del sector asistencial en salud destacan la labor del Instituto Nacional de Enfermedades Neoplásicas, el Instituto Nacional de Salud del Niño y el Instituto Nacional de Salud. Sin embargo hace falta sumar esfuerzos para poder articular y/o coordinar entre instituciones para realizar investigaciones de mayor envergadura ${ }^{(3)}$.
\end{abstract}

Es evidente que en nuestro país la medicina genómica se encuentra aún desatendida, y los esfuerzos por impulsarla son fraccionados, débiles, y con una planificación poco perceptible en las políticas sanitarias peruanas. Además, la genética y genómica médica exigen la interacción sincrónica y simbiótica de las actividades científicas y asistenciales ${ }^{(4)}$. y en el tema de financiamiento la mayoría de proyectos de esta índole logran obtener subvenciones por parte de CONCYTEC (Consejo Nacional de Ciencia, Tecnología e Innovación Tecnológica) y del FINCYT (Fondo para la Innovación, Ciencia y Tecnología), instituciones que apoyan económicamente y fomentan a que se progrese con la genómica por ahora con iniciativas aisladas, esperando que un futuro inmediato abarque otras áreas de las ciencias de la salud ${ }^{(5)}$.

En consecuencia con lo descrito anteriormente creemos que un elemento a tener en cuenta en la compleja problemática para institucionalizar esta disciplina en nuestro medio, es empezar a revisar y analizar los planes de estudio de las carreras de las ciencias biomédicas, ya que urge formar una diversidad de profesionales críticos que tienen que ver con todo el proceso de la medicina genómica, incluyendo la concepción del problema a resolver en esta temática, la puesta en marcha y/o estandarización de los protocolos más idóneos para la resolución de lo planteado, así como también el análisis de datos a través de la bioinformática para una debida interpretación, así como también la correlación y aplicación con los problemas de salud tanto a nivel individual como social sobre todo de las enfermedades más prevalentes, sin embargo llama nuestra atención un análisis preliminar realizado sobre la proporción de todas las asignaturas contempladas en los planes curriculares de las principales escuelas de medicina en nuestro país, asignaturas cuyo enfoque es fortalecer y brindar los elementos necesarios para una debida interiorización de la competencia en genómica que el

\footnotetext{
${ }^{1}$ Universidad Católica Santo Toribio de Mogrovejo, Lambayeque-Perú.

a Docente adscrito al Departamento de Ciencias de la Salud.

b Estudiante de medicina.
}

Citar como: César Ñique-Carbajal, Josely Pérez-Loaiza, Jeanette Mestanza-Quispe. Medicina genómica en las escuelas de medicina del Perú. Rev. Fac. Med. Hum. Enero 2020; 20(1):168-170. DOI 10.25176/RFMH.v20i1.2705 
futuro profesional de la medicina debería plasmar en su práctica profesional, encontrándose que el número de asignaturas que tendrían relación de manera directa e indirecta con los contenidos de la genómica, no supera el $6 \%$ del total de créditos académicos ofrecidos en la mayoría de las escuelas analizadas, tal como se muestra en la tabla 01, esta información deberá corroborarse con las competencias profesionales de los futuros médicos del Perú, con la finalidad de aplicar el avance científico y tecnológico de la genómica funcional, dado que existe complementariedad entre la medicina genómica y la mejora de la relación médico-paciente, ya que vincula a las ciencias básicas con las disciplinas clínicas, por tal motivo la enseñanza y actualización en ciencias genómicas debe considerarse prioritario ya que la concepción en el tratamiento médico está cambiando desde la medicina curativa (diagnóstico y tratamiento), medicina preventiva (intervención para evitar o retrasar la aparición de la enfermedad) a la medicina predictiva (identificación del perfil genético para un tratamiento personalizado), brindando de esta manera el soporte más certero de las enfermedades a los investigadores clínicos para identificar nuevas hipótesis relevantes de la enfermedad en beneficio de la salud de los pacientes y por consiguiente de la salud pública(6)

Tabla 1. Créditos de asignaturas relacionadas con la medicina genómica en los planes curriculares de las principales escuelas de medicina del Perú.

\begin{tabular}{|c|c|c|c|c|c|}
\hline Universidad & Régimen & $\begin{array}{l}\text { Total de } \\
\text { créditos }\end{array}$ & $\begin{array}{l}\text { Créditos } \\
\text { relacionados } \\
\text { medicina } \\
\text { genómica }\end{array}$ & Asignaturas que se imparten & $\%$ \\
\hline $\begin{array}{l}\text { Universidad Católica Santo Toribio de } \\
\text { Mogrovejo }\end{array}$ & Privado & 329 & 15 & $\begin{array}{l}\text { Biología Celular y Molecular } \\
\text { Bioquímica Genética medica }\end{array}$ & 4.5 \\
\hline Universidad Cesar Vallejo & Privado & 322 & 15 & Biología celular y hereditaria I, II y III & 4.6 \\
\hline Universidad Peruana Cayetano Heredia & Privado & 302 & 12 & $\begin{array}{l}\text { Biología Celular } \\
\text { Biología Molecular } \\
\text { Genética y embriología }\end{array}$ & 3.9 \\
\hline $\begin{array}{l}\text { Universidad Nacional Mayor de San } \\
\text { Marcos }\end{array}$ & Publico & 343 & 10 & $\begin{array}{l}\text { Bioquímica } \\
\text { Inmunología y Genética }\end{array}$ & 2.9 \\
\hline $\begin{array}{l}\text { Universidad Nacional José Faustino } \\
\text { Sánchez Carrión }\end{array}$ & Publico & 308 & 16 & $\begin{array}{l}\text { Biología Celular y Molecular } \\
\text { Bioquímica } \\
\text { Genética y embriología }\end{array}$ & 5.1 \\
\hline Universidad Nacional Federico Villareal & Publico & 308 & 16 & $\begin{array}{l}\text { Biología celular y molecular } \\
\text { Genética Humana } \\
\text { Bioquímica }\end{array}$ & 5.1 \\
\hline Universidad Ricardo Palma & Privado & 324 & 15 & $\begin{array}{l}\text { Biología celular y molecular } \\
\text { Embriología y Genética } \\
\text { Bioquímica }\end{array}$ & 4.6 \\
\hline Universidad San Martin de Porres & Privado & 336 & 17 & $\begin{array}{l}\text { Biología Celular y Molecular } \\
\text { Embriología y Genética } \\
\text { Bioquímica } \\
\text { Oncología Medica }\end{array}$ & 5 \\
\hline Universidad Nacional San Antonio Abad & Publico & 318 & 13 & $\begin{array}{l}\text { Biología Celular } \\
\text { Genética Humana } \\
\text { Bioquímica }\end{array}$ & 4 \\
\hline Universidad Católica Santa María & Privado & 304 & 16 & $\begin{array}{l}\text { Bioquímica } \\
\text { Biología Celular y Molecular } \\
\text { Bioquímica medica } \\
\text { Biología y Genética }\end{array}$ & 5.2 \\
\hline Universidad San Luis Gonzaga & Publico & 306 & 10 & $\begin{array}{l}\text { Biología } \\
\text { Embriología y Genética } \\
\text { Bioquímica }\end{array}$ & 3.2 \\
\hline Universidad Nacional Pedro Ruiz Gallo & Publico & 306 & 11 & $\begin{array}{l}\text { Biología Celular } \\
\text { Bioquímica } \\
\text { Genética }\end{array}$ & 3.5 \\
\hline Universidad Nacional San Agustín & Publico & 322 & 16 & $\begin{array}{l}\text { Bioquímica } \\
\text { Biología Molecular } \\
\text { Biología Celular y Genética }\end{array}$ & 4.9 \\
\hline
\end{tabular}


Contribuciones de autoría: Los autores participaron en la generación, recolección de información, redacción y versión final del artículo original.

Financiamiento: Autofinanciado.
Conflicto de interés: Los autores declaran no tener conflictos de interés en la publicación de este artículo.

Recibido: 26 de setiembre del 2019

Aprobado: 05 de diciembre del 2019

\section{Correspondencia: César Ñique Carbajal.}

Dirección: Av. Miguel Grau 1552 La Victoria, Chiclayo-Perú.

Teléfono: 743151561

Correo: cnique@usat.edu.pe

\section{REFERENCIAS BIBLIOGRÁFICAS}

1. Doble B, Schofield D, Roscioli T, Mattick J. Prioritising the application of genomic medicine. npj Genomic Medicine [Internet]. 2017 [Citado 02 de Octubre de 2019]; 2(35). Disponible en: https://www.nature.com/articles/ s41525-017-0037-0.pdf

2. Manolio T, Chisholm R, Ozenberger B, Roden D. Implementing genomic medicine in the clinic: the future is here. Genetics in Medicine [Internet]. 2013 April [citado 02 de Octubre de 2019]; 15(4): 258-267. Disponible en: file:///C:/ Users/user/Downloads/gim2012157a.pdf

3. Poterico J. Purisaca-Rosillo N, Taype-Rondan A. Genética y Genómica médica en el Perú. Carta al editor. Acta Med. Perú 2017; 34(2): 152-3.

4. Moran-Barrios J. Un nuevo profesional para una nueva sociedad. Respuestas desde la educación médica: la formación basada en competencias. Rev. Asoc. Esp. Neuropsiq., 2013; 33 (118), 385-405 doi: 10.4321/S021157352013000200011

5. Giono Luciana E. CRISPR/Cas9 y la terapia génica. Medicina (B. Aires) [Internet]. 2017 Oct [citado 2018 Ago 13]; 77(5): 405-409. Disponible en: $\quad$ http://www.scielo.org.ar/scielo.php?script=sci_arttext\&pid=S0025$76802017000500009 \& \operatorname{lng}=\mathrm{es}$

6. Guio H. Hacia la medicina personalizada: implicancias de las ciencias básicas y las ómicas en la práctica clínica. Rev. perú. med. exp. salud pública [Internet] 2015 [citado 21 Oct 2019]; 32(4): 629-632. Disponible en: http://www.scielo. org.pe/scielo.php?script=sci_arttext\&amp;pid=S172646342015000400001

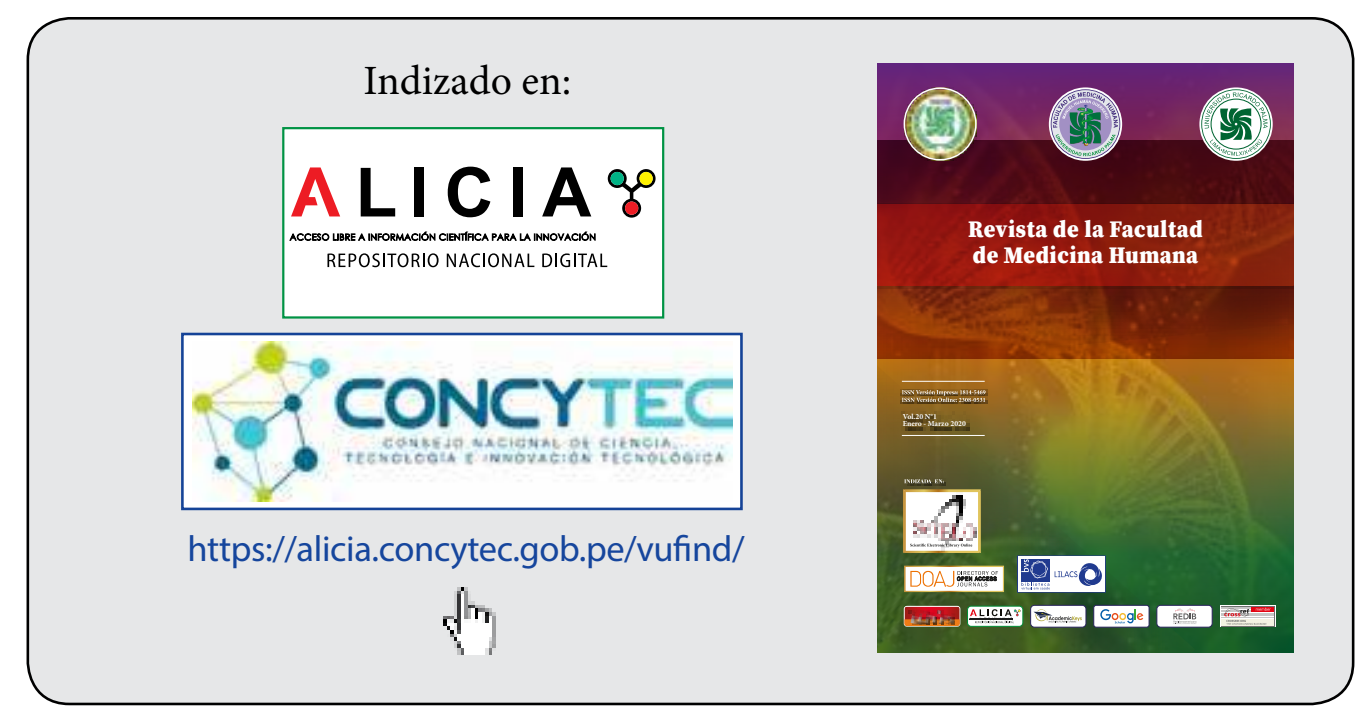

